\title{
Proteomic analysis of cerebrospinal fluid in amyotrophic lateral sclerosis
}

\author{
YAN CHEN $^{1}$, XIAO-HUI LIU ${ }^{2}$, JIAN-JUN WU ${ }^{1}$, HUI-MING REN ${ }^{1}$, \\ JIAN WANG $^{1}$, ZHENG-TONG DING ${ }^{1}$ and YU-PING JIANG ${ }^{1}$ \\ ${ }^{1}$ Department of Neurology, Huashan Hospital, Fudan University, Shanghai 200040; \\ ${ }^{2}$ Institute of Biomedical Science, Fudan University, Shanghai 200032, P.R. China
}

Received March 26, 2015; Accepted November 5, 2015

DOI: $10.3892 / \mathrm{etm} .2016 .3210$

\begin{abstract}
The present study used comparative proteomic analysis of cerebrospinal fluid (CSF) in amyotrophic lateral sclerosis (ALS) patients in order to identify proteins that may act as diagnostic biomarkers and indicators of the pathogenesis of ALS. This analysis was performed using isobaric tags for relative and absolute quantitation (iTRAQ) technology, coupled with 2-dimensional liquid chromatography/mass spectrometry. Database for Annotation, Visualization and Integrated Discovery software was utilized for bioinformatic analysis of the data. Following this, western blotting was performed in order to examine the expression of 3 candidate proteins in ALS patients compared with healthy individuals [as a normal control (NC) group] or patients with other neurological disease (OND); these proteins were insulin-like growth factor II (IGF-2), glutamate receptor 4 (GRIA4) and leucine-rich $\alpha$-2-glycoprotein 1 (LRG1). Clinical data, including gender, age, disease duration and ALS functional rating scale (ALSFRS-R) score, were also collected in the ALS patients. Multiple linear regression analysis was performed between the clinical data and the results of western blot analysis. A total of 248 distinct proteins were identified in the ALS and NC groups, amongst which a significant difference could be identified in 35 proteins; of these, 21 proteins were downregulated and 14 were upregulated. These differentially-expressed proteins were thus revealed to be associated with ALS. The western blot analysis confirmed a proportion of the data attained in the iTRAQ analysis, revealing the differential protein expression of IGF-2 and GRIA4 between the ALS and NC groups. IGF-2 was significantly downregulated in ALS patients $(\mathrm{P}=0.017)$ and GRIA4 was significantly upregulated $(\mathrm{P}=0.016)$. These results were subsequently validated in the 35-patient ALS and OND groups $(\mathrm{P}=0.002)$, but no significant difference was
\end{abstract}

Correspondence to: Dr Yu-Ping Jiang, Department of Neurology, Huashan Hospital, Fudan University, 12 Wulumuqi Zhong Road, Shanghai 200040, P.R. China

E-mail: yupingajiang@163.com

Key words: amyotrophic lateral sclerosis, cerebrospinal fluid, isobaric tags for relative and absolute quantitation, proteomics identified in LRG1 expression between these groups. GRIA4 protein expression was higher in male than female patients and was positively correlated with the ALSFRS-R score, meaning that GRIA4 expression was negatively correlated with the severity of ALS, while IGF-2 and LRG1 expression did not correlate with any clinical data. The present study thus demonstrated that GRIA4 expression levels, as a marker of severity, may be used as a reference for the timing of treatment, and that IGF-2 may serve as an effective biomarker of ALS progression.

\section{Introduction}

Amyotrophic lateral sclerosis (ALS) is a progressive neurodegenerative disease specifically affecting the upper and lower motor neurons. Due to frequent early misdiagnosis, patients do not benefit from early drug intervention and clinical drug studies have been largely unsuccessful; a correct, early diagnosis of ALS is therefore crucial.

Such a clinical diagnosis, and study of the pathogenesis of ALS, could occur through analysis of changes to the cerebrospinal fluid (CSF) proteins. Insulin-like growth factor-1, vascular endothelial growth factor, transactive response DNA-binding protein 43 , monocyte chemotactic protein 1 and other proteins have been reported as possible diagnostic indicators of ALS (1-4), but a definitive diagnostic indicator has yet to be established.

CSF quantitative proteomics, including differential in gel electrophoresis (DIGE) and isotope-coded affinity tags, have been reported in studies on Alzheimer's disease and Parkinson's disease $(5,6)$, but have not been widely used to investigate ALS. In 2005, a study by Ranganathan et al (7) was the first to investigate the CSF in ALS patients using surface-enhanced laser desorption/ionization (SELDI) technology and proteomics; three proteins, cystatin C, transthyretin and a carboxy-terminal fragment of the neuroendocrine protein 7B2, were screened and validated for their sensitivity and specificity as biomarkers. Other previous studies examined the CSF of ALS with two-dimensional gel electrophoresis, DIGE and SELDI $(8,9)$, but use of isobaric tags for relative and absolute quantitation (iTRAQ) technology in this context has not been reported, to the best of our knowledge.

The present study compared the CSF protein expression of ALS patients and healthy [normal control [NC] group) 
patients using iTRAQ labeling and 2-dimensional liquid chromatography/tandem mass spectrometry (2D LC-MS/MS) technology, screened the resulting proteins and verified their differential expression by western blotting, in order to determine the most effective biomarkers for ALS diagnosis.

\section{Patients and methods}

\section{Patients}

ALS-A group. A total of 35 patients with ALS who presented to Huashan Hospital between March 2008 and October 2010 were selected for the study. Informed consent was obtained from all patients, or their families. Tension headache sufferers were selected as the normal control (NC) group. The other neurological disease (OND) group consisted of patients who, during clinical diagnosis, were subjected to a lumbar puncture; these patients suffered from conditions such as chronic non-inflammatory peripheral neuropathy, Parkinson's disease, spastic paraplegia and hydrocephalus. Patient ages ranged between 30 and 75 years old.

$A L S-B$ group. A total of 10 cases of ALS were randomly selected from the ALS-A group and used to screen additional proteins.

CSF sample collection. Under fasting conditions, each patient was treated with the $2 \mathrm{ml} \mathrm{local}$ anesthetic lidocaine hydrochloride injection (2\%; Shanghai Harvest Pharmaceutical Co., Ltd., Shanghai, China) and subjected to a lumbar puncture, from which 8-10 ml of CSF was collected. A volume of 4-5 $\mathrm{ml}$ of CSF was immediately centrifuged at $2,000 \mathrm{x} \mathrm{g}$ for $10 \mathrm{~min}$; the resulting supernatant was collected and placed in $1.5 \mathrm{ml}$ Eppendorf tubes (Eppendorf AG, Hamburg, Germany) at $-80^{\circ} \mathrm{C}$. The remaining CSF was used for biochemical and immunological detection, as subsequently described.

Determination of protein concentration using $I T R A Q$ and $2 D L C-M S / M S$. Following the removal of 22 high-abundance proteins, including albumin and $\mathrm{IgG}$, using ProteoMiner low abundance protein enrichment kits (Bio-Rad Laboratories, Inc., Hercules, CA, USA), protein quantification was conducted using a Protein Assay reagent kit (Bio-Rad Laboratories, Inc., Hercules, CA, USA) based on Bradford methods, according to manufacturer's protocol. iTRAQ labeling was performed according to the manufacturer's protocol (Applied Biosystems Life Technologies, Foster City, CA, USA). Briefly, $100 \mu \mathrm{g}$ CSF proteins from the ALS and NC groups were precipitated with cold acetone (ratio of acetone:sample, 5:1) for $1 \mathrm{~h}$ at $-20^{\circ} \mathrm{C}$ and resuspended in $20 \mu \mathrm{l}$ dissolution buffer, respectively. Following centrifugation at 2,000 $\mathrm{x}$ g for $15 \mathrm{~min}$ and disposal of the supernatant, the precipitant was dissolved into $20 \mathrm{ul}$ iTRAQ solution and $1 \mathrm{ul} 1 \%$ sodium dodecyl sulfate (SDS). Subsequently, 1 ul cysteine sealing reagent was added for $10 \mathrm{~min}$ at room temperature. Proteins were trypsinized (Sigma-Aldrich, St. Louis, MO, USA) at $37^{\circ} \mathrm{C}$ overnight (ratio of enzyme:protein, 1:20). Peptides were labeled with iTRAQ regents for $1 \mathrm{~h}$ at room temperature. iTRAQ regents 113 and 118 were used to label the peptides from the NC and ALS groups, respectively. Following this, samples were mixed, desalted with Sep-Pak Vac C18 cartridges (Waters Corporation, Milford, MA, USA) and dried in a vacuum concentrator.
$2 D$ LC-MS/MS analysis. High-performance liquid chromatography and time-of-flight mass spectrometry (API QSTAR XL Hybrid LC-MS/MS; Applied Biosystems Life Technologies) were used for protein separation and analysis. For 2D LC-MS/MS analysis, the iTRAQ-labeled mixed peptides were fractionated using strong cation exchange (SCX) chromatography on a 20AD HPLC system (Shimadzu Corporation, Kyoto, Japan) with a polysulfoethyl column $(2.1 \times 100 \mathrm{~mm}$; $5 \mu \mathrm{m} ; 200$ Å; The Nest Group, Inc., Southborough, MA, USA). Peptide mixture was reconstituted in Buffer A (SCXA), which contained $10 \mathrm{mM} \mathrm{KH}_{2} \mathrm{PO}_{4}$ in $25 \%$ acetonitrile (pH 2.6; Thermo Fisher Scientific, Waltham, MA, USA), and loaded onto the column. Peptides were separated at a flow rate of $200 \mu \mathrm{l} / \mathrm{min}$ for $60 \mathrm{~min}$ with a gradient of $0-80 \%$ Buffer B (Buffer A supplemented with $350 \mathrm{mM} \mathrm{KCl}$ ) in Buffer A. Absorbances of $214 \mathrm{~nm}$ and $280 \mathrm{~nm}$ were identified by tandem mass spectrometry. A total of 20 SCX fractions were collected.

Protein identification. All data from tandem mass spectrometry were obtained from the UniProtKB/Swiss-Prot database using ProteinPilot 3.0 software (AB Sciex, Framingham, MA, USA), and the identification and quantification results were recorded. Search parameters were as follows: At least 1 matching peptide, a confidence interval $(\mathrm{CI})$ of the peptide of $>95 \%(\mathrm{P}<0.05)$ and results in accordance with the peak of the spectrum.

Protein annotation and classification. The Database for Annotation, Visualization and Integrated Discovery (DAVID) was used for functional annotation of proteins and gene ontology (GO) was used to classify these proteins, including their involvement in biological processes, as cellular components and their molecular function.

Differential expression of proteins. Western blotting was performed to analyze differential protein expression in the CSF between the ALS-B and NC groups, in order to verify the iTRAQ results. A total of $1 \mathrm{ml}$ CSF sample was added into a $3 \mathrm{kD}$ ultrafiltration centrifugal tube (EMD Millipore, Billerica, CA, USA) for desalination and concentration. Protein concentrations were subsequently measured via the Bradford method using Bio-Rad protein assay reagent (Bio-Rad Laboratories, Inc.). A total of $20 \mu \mathrm{g}$ protein was separated by $12 \%$ SDS polyacrylamide gel electrophoresis followed by electro-blotting onto a polyvinylidene difluoride membrane. The membrane was subsequently incubated with $5 \%$ nonfat dry milk in Tris-buffered saline at room temperature for $2 \mathrm{~h}$, in order to block non-specific binding. Following this, the membrane was incubated with the following primary antibodies: Rabbit anti-human insulin-like growth factor II (IGF-2; 1:1,250; ab9574); mouse anti-human leucine-rich $\alpha$-2-glycoprotein 1 (LRG1; 1:800; ab57992); and rabbit anti-human glutamate receptor 4 (GRIA4; 1:500; ab61171; all Abcam, Cambridge, UK), diluted in blocking buffer overnight at $4^{\circ} \mathrm{C}$. The membrane was subsequently incubated with horseradish peroxidase-conjgated AffinPure goat anti-rabbit (KC-RB-035) and anti-mouse (KC-MM-035) immunoglobulin G (H+L) secondary antibodies (both 1:5,000; Shanghai Kangcheng Biotechnology Co., Ltd., Shanghai, China) diluted with nonfat dry milk and Tris-buffered saline and Tween 20 (TBST). After rinsing three times with TBST, the western blot protein band 
Table I. Proteins analyzed in the present study.

\begin{tabular}{|c|c|c|c|c|c|}
\hline $\begin{array}{l}\text { Unused } \\
\text { ProtScore (CL, \%) }\end{array}$ & $\begin{array}{c}\text { Proteins } \\
\text { detected, } \mathrm{n}\end{array}$ & $\begin{array}{l}\text { Proteins prior to } \\
\text { grouping, } \mathrm{n}\end{array}$ & $\begin{array}{c}\text { Distinct } \\
\text { peptides, n }\end{array}$ & $\begin{array}{c}\text { Spectra } \\
\text { identified, } \mathrm{n}\end{array}$ & $\begin{array}{c}\% \text { of total } \\
\text { spectra }\end{array}$ \\
\hline$>2.0(99)$ & 211 & 285 & 18106 & 37075 & 33.8 \\
\hline$>1.3(95)$ & 248 & 347 & 19568 & 38823 & $35.4^{\mathrm{a}}$ \\
\hline$>0.47(66)$ & 294 & 448 & 21271 & 40761 & 37.2 \\
\hline
\end{tabular}

${ }^{\mathrm{a} C u t o f f}$ applied at an unused protein score of $>1.3$. CL, confidence level.

Table II. Proteins in ALS and NC groups by cerebrospinal fluid.

\begin{tabular}{|c|c|c|}
\hline Protein name & iTRAQ ratio (ALS/NC) & Accession no. \\
\hline Serum albumin & 0.9262 & sp|P02768| \\
\hline Complement C4-A & 1.0317 & sp|P0C0L4| \\
\hline Complement C3 & 1.0003 & sp|P01024| \\
\hline Transthyretin & 1.0717 & splP02766| \\
\hline$\alpha$-1-antitrypsin & 0.7250 & sp|P01009| \\
\hline$\alpha$-2-macroglobulin & 0.9938 & sp|P01023| \\
\hline Serotransferrin & 0.8150 & sp|P02787| \\
\hline Fibronectin & 1.0084 & sp|P02751I \\
\hline Apolipoprotein A1 & 1.0930 & splP02647| \\
\hline $\operatorname{Ig} \gamma 1$ chain $\mathrm{C}$ region & 0.9304 & sp|P01857| \\
\hline Apolipoprotein E & 1.1323 & sp|P02649| \\
\hline Gelsolin & 1.0509 & sp|P06396| \\
\hline Apolipoprotein A-IV & 1.1446 & splP06727| \\
\hline Clusterin & 1.0969 & splP10909| \\
\hline Cystatin C & 1.0671 & sp|P01034| \\
\hline Vitamin D-binding protein & 0.8710 & sp|P02774| \\
\hline Contactin-1 & 1.0430 & sp|Q12860| \\
\hline Complement factor & 1.0036 & sp|P08603| \\
\hline Pigment epithelium-derived factor & 0.9803 & splP36955| \\
\hline Secretogranin-1 & 1.0670 & splP05060| \\
\hline Ceruloplasmin & 0.8720 & sp|P00450| \\
\hline Serum albumin & 1.0588 & sp|P51693| \\
\hline Haptoglobin & 0.6926 & splP00738| \\
\hline Secretogranin-3 & 1.1640 & splQ8WXD2l \\
\hline Antithrombin-III & 0.8452 & splP01008I \\
\hline Chromogranin-A & 1.0098 & sp|P010645| \\
\hline$\alpha-1-B$ glycoprotein & 0.9835 & splP04217| \\
\hline$\beta$-Ala-His dipeptidase & 1.1591 & splQ96KN2l \\
\hline Neuronal cell adhesion molecule & 1.0097 & splQ92823| \\
\hline $\operatorname{Ig} \gamma 2$ chain $\mathrm{C}$ region & 1.0383 & sp|P01859| \\
\hline Monocyte differentiation antigen CD14 & 0.8775 & splP08571I \\
\hline Fibrinogen $\alpha$ chain & 1.0375 & sp|P02671I \\
\hline$\alpha$-1-antichymotrypsin & 0.9855 & sp|P01011| \\
\hline Neurosecretory protein VGF & 1.0510 & spl015240| \\
\hline$\alpha$-2-HS-glycoprotein & 1.0036 & sp|P02765| \\
\hline Angiotensinogen & 1.0014 & sp|P01019| \\
\hline $\operatorname{Ig} \alpha 1$ chain $\mathrm{C}$ region & 1.0096 & sp|P01876| \\
\hline Collagen $\alpha-1(\mathrm{I})$ chain & 1.0412 & sp|P02452I \\
\hline Plasminogen & 0.8738 & sp|P00747| \\
\hline Kininogen-1 & 0.8529 & sp|P01042I \\
\hline Fibulin-1 & 0.9324 & splP23142I \\
\hline
\end{tabular}


Table II. Continued.

Protein name

iTRAQ ratio (ALS/NC)

Accession no.

Hemoglobin subunit $\beta$

1.4623

Prostaglandin-H2 D-isomerase

0.9310

sp|P68871।

$N$-acetyllactosaminide $\beta$-1,3- $N$-acetylglucosaminyltransferase

1.0294

splP41222I

Neuronal pentraxin receptor

1.0815

splO43505|

Hemopexin

0.8432

Retinol-binding protein 4

0.9796

Apolipoprotein D

0.9616

Ectonucleotide pyrophosphatase/phosphodiesterase

0.9689

family member 2

$\beta$-2-glycoprotein 1

0.9413

splO95502।

splP02790|

spIP02753|

splP05090|

splQ13822I

Carboxypeptidase E

1.0193

Collagen $\alpha$-2(I) chain

1.0000

Calsyntenin-1

1.1224

Vitronectin

0.8401

Nucleobindin-1

1.0513

Ig $\mu$ chain $\mathrm{C}$ region

0.8467

Ig $\kappa$ chain $\mathrm{C}$ region

1.0135

Ig $\gamma 3$ chain $C$ region

0.9289

Extracellular superoxide dismutase $(\mathrm{Cu}-\mathrm{Zn})$

1.0356

Cathepsin D

0.9478

Afamin

1.0176

Complement component C7

0.9460

Apolipoprotein A-II

1.2524

Contactin-2

1.0433

Inter- $\alpha$-trypsin inhibitor heavy chain

1.0549

Neural cell adhesion molecule 1

1.0091

EGF-containing fibulin-like extracellular matrix protein

0.9392

$\operatorname{Ig} \lambda$ chain $\mathrm{C}$ regions

1.0045

Complement component C9

0.7597

Neural cell adhesion molecule L1-like protein

1.0405

Procollagen C-endopeptidase enhancer 1

1.0410

Mimecan

0.9845

Fibrinogen $\beta$ chain

1.0713

Hemoglobin subunit $\alpha$

1.5451

ProSAAS

1.0492

Neuronal pentraxin-1

1.1167

$\beta$-2-microglobulin

1.0138

Collagen $\alpha-1$ (VI) chain

1.0602

Neural cell adhesion molecule 2

0.9561

Leucine-rich $\alpha$-2-glycoprotein

0.6430

Insulin-like growth factor-binding protein 2

0.9574

Insulin-like growth factor-binding protein 6

0.9883

Protein kinase C-binding protein NELL2

0.9929

Keratin, type II cytoskeletal 1

0.9729

Dickkopf-related protein 3

1.0396

Ig $\kappa$ chain $\mathrm{V}$-III region

0.9945

Complement C1r subcomponent

0.9240

Prothrombin

0.9113

Dystroglycan

1.0292

Tetranectin

0.9282

$\alpha$-2-antiplasmin

0.9126

Complement factor B

0.8143

sp|P02749|

sp|P16870|

splP08123|

splO94985।

splP04004|

splQ02818I

splP01871I

splP01834I

sp|P01860|

splP08294|

spIP07339|

spIP43652।

sp|P10643|

spIP02652I

splQ02246।

splQ14624|

sp|P13591।

splQ12805।

spIP01842I

sp|P02748I

splO00533।

spIQ15113।

spIP20774I

splP02675।

splP69905।

splQ9UHG2I

splQ15818I

spIP61769|

spIP12109|

splO15394I

spIP02750|

spIP18065।

spIP24592।

splQ99435।

splP04264|

splQ9UBP4|

spIP01623|

splP00736I

splP00734|

splQ14118।

spIP05452।

spIP08697|

splP00751I 
Table II. Continued.

Protein name

Cartilage acidic protein 1

Peptidylglycine $\alpha$-amidating monooxygenase

Major prion protein

Zinc- $\alpha$-2-glycoprotein

Neuroendocrine protein 7B2

Multiple epidermal growth factor-like domains 8

Insulin-like growth factor-binding protein 7

SPARC

Trypsin-1

Secretogranin-2

Voltage-dependent calcium channel subunit $\alpha_{2} \delta-1$

Pyruvate kinase isozymes M1/M2

Cadherin 13

GM2 Ganglioside activator

Fibrinogen $\gamma$ chain

Extracellular matrix protein 1

Collagen $\alpha-1$ (XVIII) chain

Cadherin-2

Semaphorin 7A

Ig $\kappa$ chain V-II region GM607

Ig $\lambda$ chain V-III region LOI

Transmembrane protein $132 \mathrm{~A}$

Metalloproteinase inhibitor 2

Osteopontin

Kallikrein-6

Sex hormone-binding globulin

Actin, cytoplasmic 1

Ig $\gamma-4$ chain $C$ region

Protein FAM3C

Chorionic somatomammotropin hormone

Keratin, type I cytoskeletal 9

Limbic system-associated membrane protein

Phospholipid transfer protein

Ig heavy chain V-III region BRO

SPARC-like protein 1

Fructose-bisphosphate aldolase

$\mathrm{N}$-acetylmuramoyl-L-alanine amidase

Complement $\mathrm{C} 1$ s subcomponent

Ig $\kappa$ chain V-IV region B17

Lumican

Opioid-binding protein/cell adhesion molecule

Ribonuclease pancreatic

Ig $\kappa$ chain V-III region CLL

Immunoglobulin superfamily member 8

78-kDa glucose-regulated protein

Protein AMBP

Coagulation factor $\mathrm{V}$

Histidine-rich glycoprotein

Ig heavy chain V-III region KOL

L-lactate dehydrogenase B chain

Complement component C6

Ephrin type-A receptor 4

iTRAQ ratio (ALS/NC)

Accession no.

1.0590

0.8763

1.0478

0.7912

1.1447

0.9706

1.0327

0.8425

1.2077

0.9307

0.9343

1.0611

1.0163

1.0083

1.0925

1.0849

1.0000

1.0560

0.9433

0.9526

0.7060

1.1680

0.9855

1.0354

0.9713

0.6051

0.8566

1.1808

0.9182

0.5234

0.9161

0.9398

1.1687

0.9650

0.9325

0.9490

0.9820

0.9598

0.8581

1.0259

0.8758

0.7527

0.8486

0.8751

0.9751

0.7950

1.0938

0.9048

0.9839

0.9649

0.9164

0.9178
splQ9NQ79|

splP19021I

splP04156|

spIP25311।

splP05408I

splQ7Z7M0।

splQ16270|

splP09486|

splP07477I

spIP13521।

splP54289|

splP14618|

splP55290|

splP17900|

spIP02679|

splQ16610।

splP39060|

splP19022I

splO75326I

spIP06309|

spIP01617|

splQ24JP5।

splP16035I

spIP10451I

splQ92876।

spIP04278I

splP60709|

spIP01861।

splQ92520।

spIP01243I

spIP35527I

splQ13449|

spIP55058I

splP01766I

splQ14515।

spIP04075।

splQ96PD5l

spIP09871।

spIP06314I

spIP51884I

splQ14982।

spIP07998I

splP04207I

splQ969P0I

splP11021I

splP02760|

spIP12259|

splP04196|

spIP01772I

sp|P07195|

sp|P13671।

spIP54764| 
Table II. Continued.

Protein name

Cerebellin-3

Proenkephalin A

Insulin like growth factor binding protein 4

Apolipoprotein C-III

Trypsin -3

Transforming growth factor- $\beta$-induced protein ig-h3

IgG Fc-binding protein

Plasma serine protease inhibitor

Coagulation factor XII

Biotinidase

Ig $\kappa$ chain V-III region VG (Fragment)

Collagen $\alpha-3$ (VI) chain

Neuroserpin

Keratin, type I cytoskeletal 10

Fibulin-5

Receptor-type tyrosine-protein phosphatase $\mathrm{S}$

Complement factor I

Ig heavy chain V-III region TRO

Basement membrane-specific heparan sulfate proteoglycan core protein

$\alpha-1$ acid glycoprotein 1

Chitinase-3-like protein 1

Cell adhesion molecule 3

Galectin-3-binding protein

Ig heavy chain V-III region POM

Endonuclease domain-containing 1 protein

Ig $\lambda$ chain V-I region HA

Complement $\mathrm{C} 1 \mathrm{q}$ subcomponent subunit B

Leucine-rich repeat-containing protein 4B

Peroxiredoxin-2

Glyceraldehyde-3-phosphate dehydrogenase

Serum paraoxonase/arylesterase 1

Calcium/calmodulin-dependent protein kinase

type II $\alpha$ chain

Fibrillin-1

Complement C2

Cell growth regulator with EF hand domain protein 1

Myopalladin

Neuronal growth regulator 1

Serum amyloid A-4 protein

Protocadherin Fat 2

Cathepsin F

DNA repair protein RAD50

$\alpha$-enolase

Insulin-like growth factor II

Ig $\lambda$ chain V-III region $\mathrm{SH}$

Reelin

Pregnancy-specific $\beta$-1-glycoprotein 1

Retinoic acid receptor responder protein 2

Lymphocyte antigen $6 \mathrm{H}$

Receptor-type tyrosine-protein phosphatase N2

Multimerin-2

iTRAQ ratio (ALS/NC)

Accession no.

1.0609

1.0079

0.8461

1.1181

1.1478

1.0709

1.0775

0.9604

0.9422

1.2970

1.09987

0.9422

1.0459

0.8858

0.9587

1.1670

0.8627

1.1189

0.9080

0.7355

0.9904

0.8572

0.9876

1.0712

1.0166

1.0838

1.0301

1.0174

1.6278

1.2506

0.8635

1.1677

0.2204

0.9405

1.3740

0.6801

1.0667

1.0645

1.1409

1.1142

0.9463

1.1591

0.4053

1.0399

1.1149

0.7522

1.0850

1.0322

1.0020

1.0029
splQ6UW01

spIP01210|

spIP22692I

splP02656I

splP35030|

splQ15582।

splQ9Y6R7I

splP05154I

splP00748I

splP43251I

splP04433|

splP00748I

splQ99574I

spIP13645।

splQ9UBX5।

splQ13332।

splP05156I

spIP01762I

splP98160|

spIP02763|

spIP36222I

splQ8N126l

splQ08380।

splP01774I

sp|P01776I

sp|P01779|

sp|P02746|

spIQ9NT99|

splP32119|

splP04406I

splP27169|

splQ9UQM7|

sp|P35555।

spIP06681I

splQ99674I

splQ86TC9|

splQ7Z3B1l

spIP35542I

spIQ9NYQ8I

splQ9UBX1।

splQ92878I

spIP06733I

spIP01344I

splP01714

spIP78509|

sp|P11464|

splQ99969|

splO94772।

splQ92932I

spIQ9H8L6I 
Table II. Continued.

\begin{tabular}{|c|c|c|}
\hline Protein name & iTRAQ ratio (ALS/NC) & Accession no. \\
\hline Apolipoprotein L1 & 0.9537 & splO14791| \\
\hline Ig $\kappa$ chain V-I region Roy & a & splP01608| \\
\hline Neurofascin & 1.0305 & splO94856| \\
\hline V-type proton ATPase & 0.8780 & splQ15904| \\
\hline Heparin cofactor 2 & 1.0087 & splP05546| \\
\hline Plasma glutamate carboxypeptidase & 1.0663 & splQ9Y646| \\
\hline Hypoxia upregulated protein 1 & 1.0213 & splQ9Y4L1| \\
\hline Ig $\kappa$ chain $\mathrm{V}$-I region $\mathrm{Ka}$ & 0.9834 & splP01603| \\
\hline Protein DJ-1 & 1.2886 & splQ99497| \\
\hline Laminin subunit $\gamma-1$ & 0.8128 & sp|P11047| \\
\hline Cell surface glycoprotein MUC18 & 0.7681 & sp|P43121I \\
\hline Neuroendocrine convertase 2 & 1.2290 & sp|P16519| \\
\hline Inter- $\alpha$-trypsin inhibitor heavy chain H5 & 0.9165 & splQ86UX2| \\
\hline Exostosin-like 2 & 0.9342 & splQ9UBQ6| \\
\hline Metalloproteinase inhibitor 1 & 1.0673 & sp|P01033| \\
\hline Immunoglobulin $\mathrm{J}$ chain & 1.0429 & sp|P01591| \\
\hline Ig $\kappa$ chain $\mathrm{V}$-I region $\mathrm{BAN}$ & a & splP04430| \\
\hline Ig $\kappa$ chain V-I region DEE & 1.0241 & splP01597| \\
\hline Ig $\kappa$ chain V-I region Wes & 0.8814 & splP01611l \\
\hline Serum amyloid A-1 protein & 0.6516 & splP02735| \\
\hline Glutamate receptor 4 & 1.3098 & sp|P48058| \\
\hline Amyloid $\beta$ A4 & 1.0164 & sp|P05067| \\
\hline Zinc finger protein & 0.9751 & splB1APH4| \\
\hline Nidogen-2 & 1.0441 & splQ14112| \\
\hline 72-kDa type IV collagenase & 0.8378 & sp|P08253| \\
\hline WAP, kazal, immunoglobulin, Kunitz and & 1.0204 & splQ8TEU8I \\
\hline \multicolumn{3}{|l|}{ NTR domain-containing protein 2} \\
\hline Kallistatin & 0.8933 & sp|P29622| \\
\hline 45-kDa calcium-binding protein & 1.0575 & splQ9BRK5l \\
\hline Tissue $\alpha$-L-fucosidase & 1.1211 & sp|P04066| \\
\hline protein Cut A & 1.0521 & splO60888| \\
\hline Ig heavy chain V-I region & 0.9126 & sp|P06326| \\
\hline Ig heavy chain V-I region & 0.9126 & sp|P06326| \\
\hline$\gamma$-glutamyl hydrolase & 1.2209 & splQ92820| \\
\hline Complement component $\mathrm{C} 8 \gamma$ chain & 0.9202 & sp|P07360| \\
\hline Phosphatidylethanolamine-binding protein 1 & 1.1293 & sp|P30086| \\
\hline Thy-1 membrane glycoprotein & 0.7535 & splP04216| \\
\hline Cell adhesion molecule 4 & 0.9868 & splQ8NFZ8| \\
\hline Sjoegren syndrome/scleroderma autoantigen 1 & 0.9615 & splO60232| \\
\hline Uncharacterized protein C6orf170 & 1.1061 & splQ96NH3| \\
\hline$N$-acetylglucosamine-1-phosphotransferase subunit $\gamma$ & 1.0938 & splQ9UJJ9| \\
\hline Testican-2 & 1.2140 & splQ92563| \\
\hline Fructose-bisphosphate aldolase C & a & sp|P09972| \\
\hline Lysozyme C & 0.8222 & sp|P61626| \\
\hline V-type proton ATPase subunit D & 1.2915 & splQ9Y5K81 \\
\hline Coagulation factor XI & a & sp|P03951| \\
\hline Complement $\mathrm{C} 1 \mathrm{q}$ subcomponent subunit $\mathrm{C}$ & 0.8441 & sPl02747| \\
\hline Dermcidin & 0.7257 & sp|P81605| \\
\hline Ig $\kappa$ chain V-II region RPMI 6410 & 0.7960 & sp|P06310| \\
\hline Hemoglobin subunit $\delta$ & a & splP06310| \\
\hline Titin & 0.9960 & splQ8WZ42| \\
\hline Tumor protein 63 & 0.7445 & splQ9H3D4| \\
\hline
\end{tabular}


Table II. Continued.

\begin{tabular}{lcc}
\hline Protein name & iTRAQ ratio (ALS/NC) & Accession no. \\
\hline Cysteine-rich with EGF-like domain protein 1 & 1.0219 & splQ96HD1l \\
Putative $\alpha-1$-antitrypsin-related protein & 0.8877 & splP20848| \\
Scrapie-responsive protein 1 & 1.0576 & splO75711।
\end{tabular}

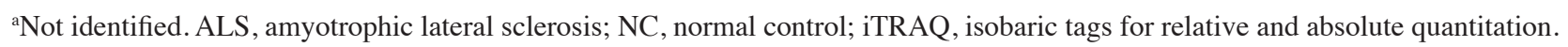

Table III. Proteins decreased in ALS group.

\begin{tabular}{|c|c|c|}
\hline Protein & Ratio of ALS vs. control & Accession no. \\
\hline$\alpha$-1-antitrypsin $\alpha 1$ & 0.7250 & sp|P01009| \\
\hline Haptoglobin & 0.6926 & sp|P00738| \\
\hline Complement component 9 & 0.7597 & splP02748| \\
\hline Leucine-rich $\alpha$-2-glycoprotein & 0.6430 & splP02750| \\
\hline Zinc- $\alpha$-2-glycoprotein & 0.7912 & sp|P25311| \\
\hline Sex hormone-binding globulin & 0.6051 & splP04278I \\
\hline Chorionic somatomammotropin hormone 1 & 0.5234 & sp|P01243| \\
\hline Ribonuclease pancreatic & 0.7527 & splP07998| \\
\hline Protein AMBP & 0.7950 & splP02760| \\
\hline$\alpha$-1-acid glycoprotein 1 & 0.7355 & sp|P02763| \\
\hline Fibrillin-1 & 0.2204 & sp|P35555| \\
\hline Myopalladin & 0.6801 & splQ86TC9| \\
\hline Insulin-like growth factor II & 0.4053 & sp|P01344| \\
\hline Pregnancy-specific $\beta$-1-glycoprotein 1 & 0.7522 & splP43251I \\
\hline Cell surface glycoprotein MUC18 & 0.7681 & sp|P43121I \\
\hline Serum amyloid A protein & 0.6516 & sp|P02735| \\
\hline Thy-1 membrane glycoprotein & 0.7535 & sp|P04216| \\
\hline Dermcidin & 0.7257 & sp|P81605| \\
\hline Ig $\lambda$ chain V-III region LOI & 0.7060 & sp|P01617| \\
\hline Ig $\kappa$ chain V-II region RPMI 6410 & 0.7960 & splP06310| \\
\hline Tumor protein 63 & 0.7444 & splQ9H3D4| \\
\hline
\end{tabular}

ALS, amyotrophic lateral sclerosis.

was detected using chemiluminescence, and the gray scales of the bands were quantified using software Image Lab 3.0 (Bio-Rad Laboratories, Inc.).

Statistical analysis. SPSS17.0 (SPSS, Inc., Chicago, IL, USA) was used for statistical analyses, GraphPad Prism 4 (GraphPad Software, Inc., La Jolla, CA, USA) was used to draw graphs and ProteinPilot 3.0 was used to detect the protein threshold [where Unused ProtScore $>1.3$ (95\% CI)]. An error (ProtScore) of 2.0 indicated a credible identified protein; an error of $>1.2$ or $<0.8$ indicated an identifiable significant difference $(\mathrm{P}<0.05)$.

All data were normally distributed when examined with a one-sample Kolmogorov-Smirnov test. A t-test was used to compare two groups and data are expressed as the mean \pm standard deviation; $\mathrm{P}<0.05$ was considered to indicate a statistically significant difference.
Correlation analysis used multiple linear regression analysis and the disaggregated data was assigned a conversion score, as follows: i) Gender: male, 1; and female, 2; ii) diagnostic level: diagnosed, 1; suspected, 2; suspected and clinically supported, 3; iii) involvement: medullary, 1; cervical, 2; and lumbar, 3.

\section{Results}

Clinical data. The average ages of the ALS-B and NC groups were $52.7 \pm 12.13$ and $51.1 \pm 10.62$ years old, respectively, and there were 6 men and 4 women in each group. No significant difference in age or gender balance between these groups was identified $(\mathrm{P}>0.05)$.

The average ages of the ALS-A and OND groups were $52.80 \pm 11.98$ and $51.17 \pm 12.44$ years old, respectively, and there were 22 men and 13 women in the ALS-A group, 
Table IV. Increased proteins in ALS group.

\section{Protein}

Ratio of ALS vs. control

Accession no.

Peroxiredoxin-2

1.6278

sp|P32119|

Glutamate receptor 4

1.3097

splP02735|

Apolipoprotein A-II

1.2523

splP48058|

Hemoglobin subunit $\alpha$

1.5451

sp|P69905।

Trypsin-1

1.2076

splP69905।

Biotinidase

1.2970

splP43251I

Hemoglobin subunit $\beta$

1.4623

splP68871I

Glyceraldehyde-3-phosphate dehydrogenase

1.2505

splP04406|

Cell growth regulator with $\mathrm{EF}$ hand domain protein 1

1.3748

splQ99674I

Protein DJ-1

1.2886

splQ99497I

Neuroendocrine convertase 2

1.2294

splP16519|

$\gamma$-glutamyl hydrolase

1.2209

splQ92820|

Testican-2

1.2140

splQ92563।

V-type proton ATPase subunit D

1.2915

ALS, amyotrophic lateral sclerosis.
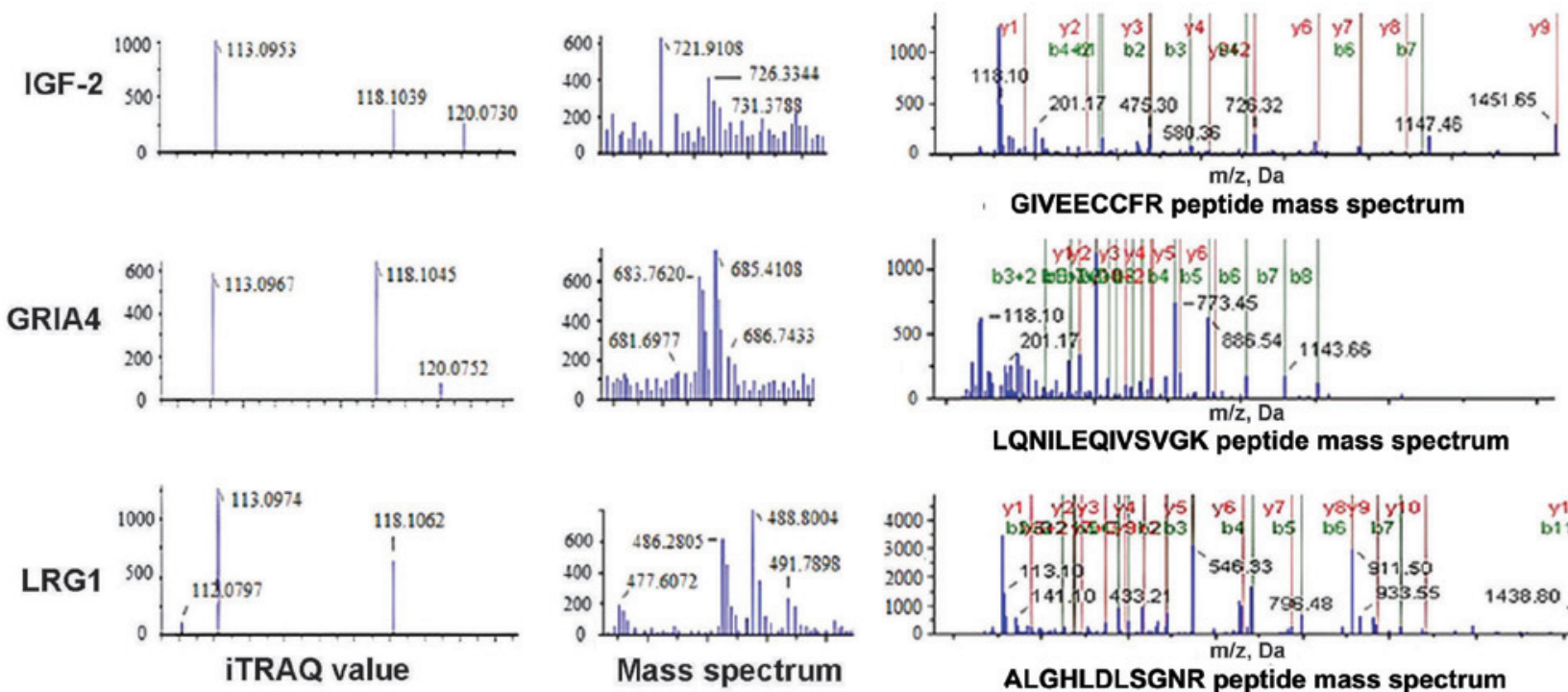
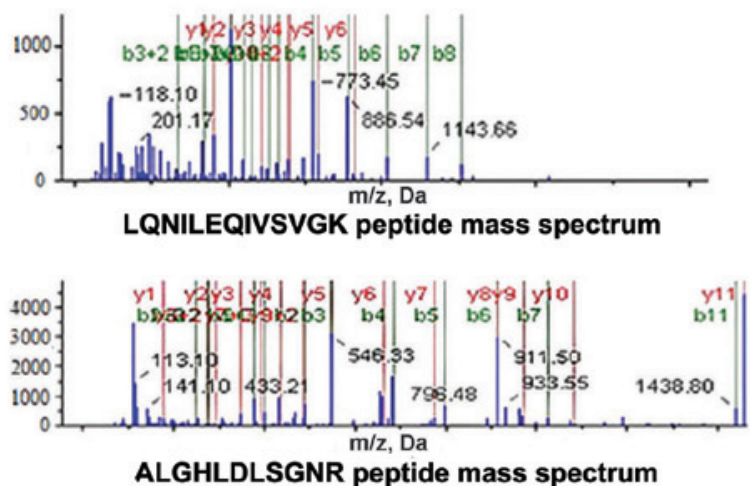

Figure 1. Sample data of 3 differentially-expressed proteins. GIVEECCFR, ALGHLDLSGNR and LQNILEQIVSVGK are enzyme-specific peptides. IGF-2, insulin-like growth factor II; GRIA4, glutamate receptor 4; LRG1, leucine-rich $\alpha$-2-glycoprotein 1; iTRAQ, isobaric tags for relative and absolute quantitation.

and 11 men and 7 women in the OND group. No significant difference was identified in age or gender balance between these groups $(\mathrm{P}>0.05)$. The protein concentration of CSF was $350.46 \pm 110.09 \mathrm{mg} / \mathrm{l}$ in the ALS-A group and $377.56 \pm 85.85 \mathrm{mg} / \mathrm{l}$ in the control group, with no significant difference revealed between the two $(\mathrm{P}>0.05)$.

CSF protein identification. iTRAQ and 2D-LC-MS/MS analyses were performed and used to analyze the protein content of the CSF in the ALS and NC groups. A total of 248 proteins were identified, and their names, the iTRAQ ratio (where available) and the UniProtKB/Swiss-Prot database accession number of 243 of these proteins are provided $(95 \% \mathrm{CI}$; Tables I and II).
Analyses of differential protein expression. A total of 35 differentially-expressed proteins were compared between the ALS and NC groups; of these, 14 were upregulated and 21 were downregulated (Tables III and IV). These proteins had a ProtScore between the values of $>1.2$ and $<0.8$, corresponding to $\mathrm{P}<0.05$.

Sample data of specific differentially-expressed proteins. IGF-2 and LRG1 protein expression was decreased in the experimental groups, whereas GRIA4 expression was increased (Fig. 1).

DAVID results and the classification of proteins by biological role. The function of all identified proteins was analyzed using 


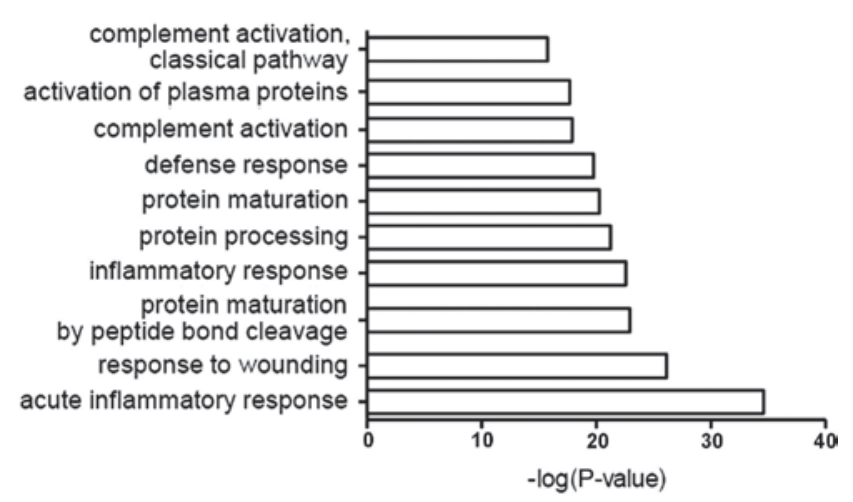

Figure 2. Identified cerebrospinal fluid proteins, classified by the biological processes that they are involved in. Activation of plasma proteins refers to this process in the acute inflammatory response.

GO in conjunction with DAVID software. The most common biological roles of CSF proteins were in acute inflammation, damage response, protein maturation, inflammation, defense response, complement activation and other associated immune pathways (Fig. 2).

Classification by cellular localization. The most common localization of CSF proteins relative to cells included the extracellular domain, extracellular space, extracellular matrix and protein-lipid complexes (Fig. 3).

Classification by molecular function. The most common molecular functions of CSF proteins were endopeptidase, peptidase, enzyme and serine-type endopeptidase inhibitors, and antigen-, calcium- and heparin-binding proteins (Fig. 4).

Western blotting. A total of 3 candidate proteins were randomly selected to be examined by western blot analysis in the ALS and the NC groups (Fig. 5); of these, IGF-2 was revealed to be significantly downregulated and GRIA4 was significantly upregulated in the ALS group when compared with the normal control group $(\mathrm{P}<0.05$; Table $\mathrm{V})$, but LRG1 expression was not significantly altered $(\mathrm{P}=0.224$; Table $\mathrm{V})$. These proteins were also examined by western blot analysis in the ALS-A and OND groups, again demonstrating a significant downregulation of IGF-2 and a significant upregulation of GRIA4 in the ALS group compared with the OND group $(\mathrm{P}<0.01$; Table $\mathrm{VI})$, but no significant difference in LRG1 expression between these groups $(\mathrm{P}=0.196$; Table VI).

Correlation between GRIA4 and gender. GRIA4 expression in the ALS-A group was significantly higher in male patients than in female patients $(765,483 \pm 583,227$ and $319,766 \pm 224,242$, respectively; r=-0.574; $\mathrm{P}=0.003$; Fig. 6).

GRIA4 expression in the ALS-A group was also positively correlated with ALS clinical scores $(r=0.487 ; \mathrm{P}=0.017)$, indicating a negative correlation with clinical severity (Fig. 7).

\section{Discussion}

In the present study, 248 different low-abundance proteins were identified in human CSF and the details of these proteins were established in ALS patients. All proteins were subjected

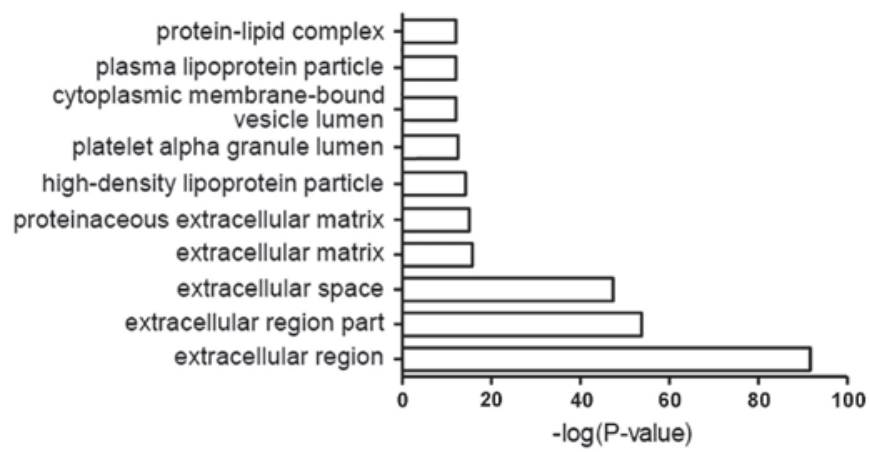

Figure 3. Identified cerebrospinal fluid proteins, classified by their cellular localization. Extracellular region refers to the space external to the outermost structure of the cell, indicating gene products that are not attached to the cell surface. Extracellular region part refers to any constituent part of the extracellular region, and is not used to specifically indicate gene products.

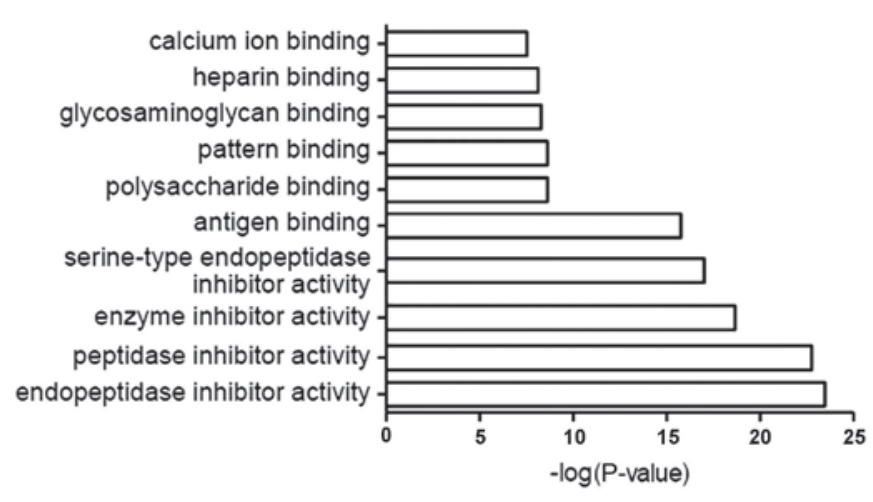

Figure 4. Identified cerebrospinal fluid proteins, classified by their molecular function.

to GO analysis with DAVID software and were classified according to their involvement in biological processes, their cellular localization and their molecular function. Data indicated that the primary roles of these proteins were in the acute inflammatory response and injury response, that the proteins were predominantly localized to extracellular regions and that the majority of these proteins were endopeptidase and peptidase inhibitors. These data aid the understanding of CSF protein profiles in patients with ALS, and provide possible biomarkers of the disease. A screening of 35 of these proteins revealed significant differences in protein expression between the ALS and NC groups, primarily in inflammation-associated proteins, neurotrophic factors and signal transduction proteins.

IGF-2, GRIA4 and LRG1 were randomly selected to verify their differential expression in ALS patients using western blot analysis. Consistent with the results of the proteomic analysis, IGF-2 and GRIA4 expression was altered in the CSF of ALS patients, but there was no significant difference in LRG1 expression between the ALS and NC groups; this led to the conclusion that additional verification of the altered protein expression reported in the present study is necessary to confirm these proteomic results.

To confirm the expression specificity of IGF-2, GRIA4 and LRG1, expression levels of these proteins were compared in patients with ALS and patients with OND; IGF-2 expression 
Table V. Western blotting results of ALS-B and NC groups.

\begin{tabular}{lcccc}
\hline Protein & Molecular weight, KDa & ALS group $(\mathrm{n}=10)$ & NC group $(\mathrm{n}=10)$ & P-value \\
\hline IGF-2 & 7.5 & $225700 \pm 126090$ & $436857 \pm 212550$ & $0.017^{\mathrm{a}}$ \\
GRIA4 & 102 & $715730 \pm 432220$ & $305796 \pm 130600$ & $0.016^{\mathrm{a}}$ \\
LRG1 & 38 & $1278000 \pm 702040$ & $1807000 \pm 1115500$ & 0.224 \\
\hline
\end{tabular}

Data are presented as the mean \pm standard deviation. ${ }^{a} \mathrm{P}<0.05$ vs. $\mathrm{NC}$ group. ALS, amyotrophic lateral sclerosis; $\mathrm{NC}$, normal control; IGF-2, insulin-like growth factor II; GRIA4, glutamate receptor 4; LRG1, leucine-rich $\alpha$-2-glycoprotein 1.

Table VI. Western blotting results of ALS-A and OND groups.

\begin{tabular}{lrrr}
\hline Protein & ALS group $(\mathrm{n}=35)$ & OND group $(\mathrm{n}=18)$ & P-value \\
\hline IGF-2 & $222200 \pm 123648$ & $452500 \pm 255620$ & $0.002^{\mathrm{a}}$ \\
GRIA4 & $608502 \pm 519012$ & $200100 \pm 150810$ & $0.002^{\mathrm{a}}$ \\
LRG1 & $1097255 \pm 961025$ & $746070 \pm 703690$ & 0.196 \\
\hline
\end{tabular}

Data are presented as the mean \pm standard deviation. ${ }^{a} \mathrm{P}<0.01$ vs. OND group. ALS, amyotrophic lateral sclerosis; OND, other neurological disease; IGF-2, insulin-like growth factor II; GRIA4, glutamate receptor 4; LRG1, leucine-rich $\alpha$-2-glycoprotein 1.
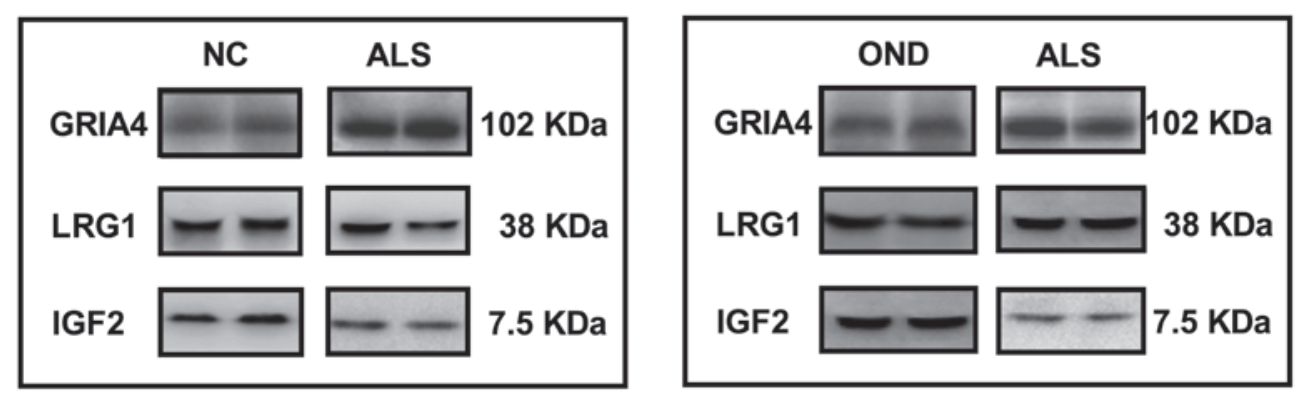

Figure 5. Western blot analysis of the three candidate proteins, glutamate receptor 4 (GRIA4), leucine-rich $\alpha$-2-glycoprotein 1 (LRG1) and insulin-like growth factor II (IGF-2). NC, normal control; ALS, amyotrophic lateral sclerosis; OND, other neurological disease.



Figure 6. Correlation between GRIA4 and clinical features. GRIA4, glutamate receptor 4 .

was significantly decreased, but GRIA4 expression was significantly increased.

Alterations to protein expression are complex with regard to disease progression, age, gender and duration of illness; it was thus important to examine the correlation between alterations to protein expression and clinical features. Clinical data of 35 ALS patients was collected and were subjected to multiple linear regression analysis to reveal any confounding factors.



Figure 7. Correlation of ALS value with GRIA4. ALS, amyotrophic lateral sclerosis; GRIA4, glutamate receptor 4; ALSFRS, ALS functional rating scale.

The clinical data in the present study revealed a higher male incidence of ALS (male to female ratio, 1.7:1), which was in support of a previous study; the 2009 European epidemiological study revealed a similar ratio of 1.4:1 (10). The present results demonstrated a correlation of GRIA4 expression with 
gender; male GRIA4 levels were 2.5-fold those of female levels $(\mathrm{P}<0.01)$.

To the best of our knowledge, the association between glutamate receptor levels and clinical characteristics has not been studied; however, glutamate excitotoxicity damage is widely recognized in the pathogenesis of ALS. Fiszman et al (11) reported no significant correlation between glutamate ligand concentration in the CSF of patients with different severities of ALS, suggesting that glutamate is involved in the occurrence of ALS and not in the severity of the disease. Excitotoxicity of glutamate also requires the presence of a glutamate receptor, meaning that high expression of glutamate receptors may be responsible for the neuronal toxicity injury induced by glutamate. As the concentration of glutamate is increased in the CSF of ALS patients (11), and GRIA4 expression was increased in ALS in the current study, the high incidence of ALS may be associated with the expression of GRIA4.

In the present study, the ALS score was estimated using the ALSFRS-R scale; a lower score on this scale corresponded to more severe disease. A multivariate analysis indicated that GRIA4 expression was positively correlated with the ALS score, revealing a negative correlation with the severity of the disease. However, ALS patients with mild symptoms were selected, defined in accordance with a previous scoring system attributing a score $>25$ to less severe ALS and scores of $<25$ to moderate and severe phases of ALS (12). As the glutamate concentration is significantly increased in the CSF of ALS patients (7), glutamate is likely to be involved in the pathogenesis of the disease. From the present results, it was concluded that GRIA4 expression is likely to be involved in the pathogenesis of ALS, resulting in a negative feedback regulatory mechanism to subsequently reduce its expression. The glutamate receptor antagonist, riluzole, is effective in the early treatment of ALS (13). In conjunction with the present report suggesting the early-stage overexpression of GRIA4, these data indicate that early treatment with anti-glutamate-associated drugs may prove a useful therapeutic measure.

The multivariate analysis examining IGF-2 and LRG1 expression and the clinical data revealed no significant correlations. This may be attributable to the sample size of the present study being too small or too few clinical factors being included. Based on the standard deviation values, the expression levels of IGF-2 and LRG1 were relatively balanced, as compared with the standard deviation of the GRIA4 expression levels, which suggested that IGF-2 may be a valuable biomarker of ALS with higher credibility due to fewer interference factors.

In summary, GRIA4 expression varied based on gender and may be reflective of ALS severity, providing a meaningful reference value for the timing of treatment. Furthermore, IGF-2 may prove an effective diagnostic marker of ALS.

\section{Acknowledgements}

The present study was supported by the Scientific Research Foundation of Huashan Hospital, Fudan University (Dr Yan Chen; 2007). The authors would like to thank staff from the Institute of Biomedical Science (Fudan University, Shanghai, China) for providing technical support.

\section{References}

1. Corbo M, Lunetta C, Magni P, Dozio E, Ruscica M, Adobbati L and Silani V: Free insulin-like growth factor (IGF)-1 and IGF-binding proteins-2 and -3 in serum and cerebrospinal fluid of amyotrophic lateral sclerosis patients. Eur J Neurol 17: 398-404, 2010

2. Devos D, Moreau C, Lassalle P, Perez T, De Seze J, Brunaud-Danel V, Destée A, Tonnel AB and Just N: Low levels of the vascular endothelial growth factor in CSF from early ALS patients. Neurology 62: 2127-2129, 2004.

3. Kasai T, Tokuda T, Ishigami N, Sasayama H, Foulds $P$ Mitchell DJ, Mann DM, Allsop D and Nakagawa M: Increased TDP-43 protein in cerebrospinal fluid of patients with amyotrophic lateral sclerosis. Acta Neuropathol 117: 55-62, 2009.

4. Nagata T, Nagano I, Shiote M, Narai H, Murakami T, Hayashi T, Shoji M and Abe K: Elevation of MCP-1 and MCP-1/VEGF ratio in cerebrospinal fluid of amyotrophic lateral sclerosis patients. Neurol Res 29: 772-776, 2007.

5. Zhang J, Goodlett DR, Quinn JF, Peskind E, Kaye JA, Zhou Y, Pan C, Yi E, Eng J, Wang Q, et al: Quantitative proteomics of cerebrospinal fluid from patients with Alzheimer disease. J Alzheimers Dis 7: 125-133, discussion 173-180, 2005.

6. Jin J, Meredith GE, Chen L, Zhou Y, Xu J, Shie FS, Lockhart P and Zhang J: Quantitative proteomic analysis of mitochondrial proteins: Relevance to Lewy body formation and Parkinson's disease. Brain Res Mol Brain Res 134: 119-138, 2005.

7. Ranganathan S, Williams E, Ganchev P, Gopalakrishnan V, Lacomis D, Urbinelli L, Newhal K, Cudkowicz ME, Brown RH Jr and Bowser R: Proteomic profiling of cerebrospinal fluid identifies biomarkers for amyotrophic lateral sclerosis. J Neurochem 95: 1461-1471, 2005.

8. Ryberg H, An J, Darko S, Lustgarten JL, Jaffa M, Gopalakrishnan V, Lacomis D, Cudkowicz M and Bowser R: Discovery and verification of amyotrophic lateral sclerosis biomarkers by proteomics. Muscle Nerve 42: 104-111, 2010.

9. Ekegren T, Hanrieder J and Bergquist J: Clinical perspectives of high-resolution mass spectrometry-based proteomics in neuroscience: Exemplified in amyotrophic lateral sclerosis biomarker discovery research. J Mass Spectrom 43: 559-571, 2008.

10. Logroscino G, Traynor BJ, Hardiman O, Chiò A, Mitchell D, Swingler RJ, Millul A, Benn E and Beghi E; EURALS: Incidence of amyotrophic lateral sclerosis in Europe. J Neurol Neurosurg Psychiatry 81: 385-390, 2009.

11. Fiszman ML, Ricart KC, Latini A, Rodríguez G and Sica RE: In vitro neurotoxic properties and excitatory aminoacids concentration in the cerebrospinal fluid of amyotrophic lateral sclerosis patients. Relationship with the degree of certainty of disease diagnoses. Acta Neurol Scand 121: 120-126, 2010.

12. Iłzecka J: Cerebrospinal fluid Flt3 ligand level in patients with amyotrophic lateral sclerosis. Acta Neurol Scand 114: 205-209, 2006.

13. Miller RG, Mitchell JD, Lyon M and Moore DH: Riluzole for amyotrophic lateral sclerosis (ALS)/motor neuron disease (MND). Cochrane Database Syst Rev 1: CD001447, 2007. 